\title{
A NEW PLANT MODELLING APPROACH FOR FORMAL VERIFICATION PURPOSES
}

\author{
José Machado*, Eurico Seabra*, Filomena Soares ${ }^{* *}$, José Campos ${ }^{* * *}$ \\ ${ }^{*}$ Mechanical Engineering Department, ${ }^{* *}$ Electronics Engineering Department, ${ }^{* * *}$ \\ Informatics Department; School of Engineering; University of Minho, Campus of \\ Azurém, 4800-058 Guimarães, PORTUGAL
}

\begin{abstract}
This paper presents a new approach in plant modeling for the formal verification of real time systems. A system composed by two tanks is used, where all its components are modeled by simple modules and all the interdependences of the system's modular models are presented. As innovating parameters in the plant modeling, having as purpose its use on formal verification tasks, the plant is modeled using Dymola software and Modelica programming language. The results obtained in simulation are used to define the plant models that are used for the formal verification tasks, using the model-checker UPPAAL. The paper presents, in a more detailed way, the part of this work that is related to formal verification, being pointing out the used plant modeling approach. Copyright $\mathbb{C} 2007$ IFAC
\end{abstract}

Keywords: Safe Systems, Real Time Systems, Plant Models, Formal Verification

\section{INTRODUCTION}

Safety control has, as its main goal, the assurance of the requirements of reliability, availability, and maintainability of automated systems ${ }^{1}$. Because of its direct impact on people and goods safety, the reliability of critical systems (transports, space, nuclear,...) has, since some time, mobilized the scientific community efforts. To assure the safety of a system, it is necessary to use a global approach (to guarantee that weaknesses do not exist) that takes into account a set of engineering activities and, later, after entering into functioning, the set of activities of exploitation and maintainability under conditions of operational exploitation of the system. Thus, the

\footnotetext{
${ }^{1}$ This research project is carried out in the context of the SCAPS Project supported by FCT, the Portuguese Foundation for Science and Technology, and FEDER, the European regional development fund, under contract $\mathrm{POCI} / \mathrm{EME} / 61425 / 2004$ that deals with safety control of automated production systems.
}

scientific community makes an effort to find, for the engineers, models, methods and tools, having as complementary objectives to anticipate possible predictions of malfunctioning.

Among the several techniques of industrial controllers analysis there are distinguished, for its utility, Simulation (Baresi et al. 2000) and Formal Verification (Moon, 1994). In the research works on industrial controllers analysis, these two techniques are rarely used simultaneously. If Simulation is faster to execute, has the limitation of considering only some system behavior evolution scenarios. Using Formal Verification has the advantage of testing all the possible system behavior evolution scenarios but, sometimes, it has the limitation of the time necessary for the attainment of formal verification results. This paper shows, how it is possible, and desirable, to conciliate these two techniques in the analysis of industrial controllers. With the simultaneous use of these two techniques, the developed industrial controllers are more robust and not subject to errors. 
This paper is focused on the formal verification of timed systems.

There are several approaches to applying formal verification techniques (not considering timed aspects) on automation systems dependability: from the formal verification by theorem proving (Roussel and Denis, 2002) to formal verification by modelchecking (Rossi, 2004) and considering (Machado et al. 2006), or not (Rossi, 2004), a plant model. In the formal verification of timed systems there are several approaches to increase the obtained results in proving properties. Between these works there are distinctions between the work by (Remelhe et al. 2004), where the translation of IEC 61131-3 SFC into timed automata is studied, and the work by (Gaid et al. 2005) where a way for constructing the controller model is proposed, using the initial approach proposed by (Mader and Wupper, 1999), and some work hypotheses related with the controller model evolution and with time aspects, that increase the obtained results on formal verification tasks.

In this work we are adopting the base system and the work hypothesis considered by (Gaid et al. 2005) for the controller behavior and we propose a way to build plant timed models. To achieve our goals, in this work, the paper is organized as follows. Section 1 , presents the challenge proposed in this work. Section 2 is devoted to the general presentation of the case study involving a system with two tanks, a heating device, level control sensors and valves to control the liquids flow and it presents also the methodology to obtain the controller program deduced from a specification of the system desired behavior. Section 3 is entirely dedicated to plant modeling, being presented the adopted approach. Next (section 4) a set of system behavior properties to prove is provided and also its formalization using TCTL. Section 5 presents and discusses the obtained results on formal verification using the modelchecker UPPAAL and, finally, section 6, presents some conclusions and future work.

\section{CASE STUDY}

In this work a modified version of the benchmark example for an behavior evaporator system presented by (Kowalewski et al. 2001) and (Huuck et al. 2001) is used. The system (Fig. 1) consists of two tanks (one of them is heated and mixed), a condenser, level sensors and on-off valves (Vi). In the normal operation mode the system works as follows. Tank1 is filled with two solutions by opening valves $\mathrm{V} 1$ and V2. Then, the mixer starts working in order to promote the dilution. After two time units, the heated device is switched on for 20 time units to increase temperature solution. During this period part of the liquid is evaporated and cooled by the condenser. At that point the required liquid concentration has been reached and the heater is switched off. The remaining liquid is drained to tank 2 by opening valve V3. The mixing device is switched off when tank1 is empty. The solution stays in tank 2 for post-processing, to stay liquid, for 32 time units and then valve V4 is open to empty tank 2 .

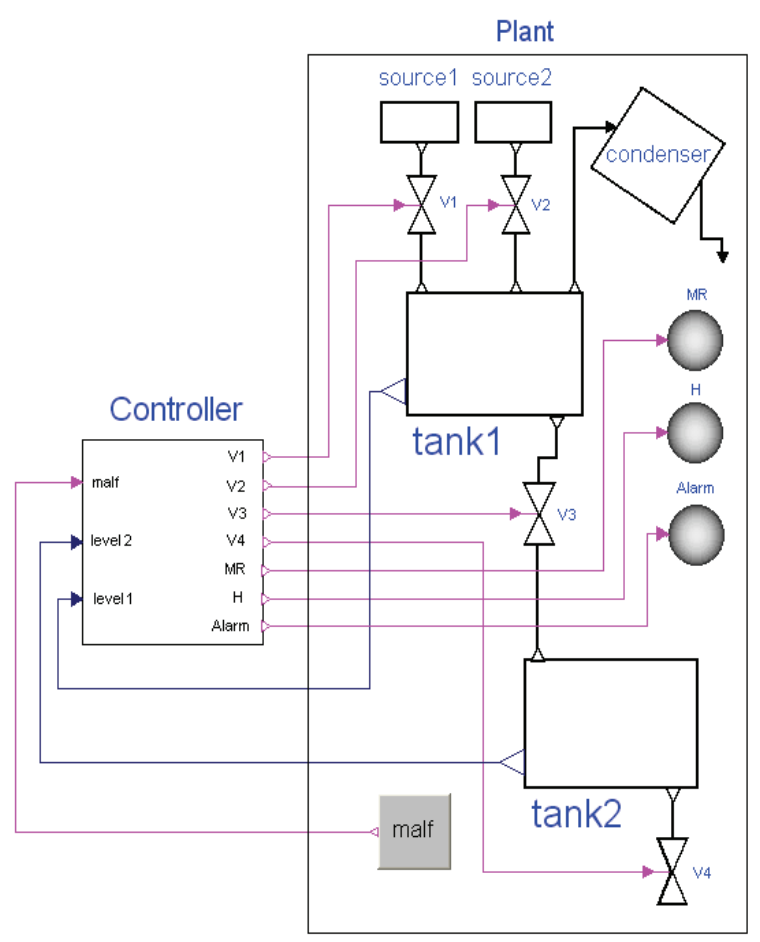

Fig. 1. Evaporator system. Closed-loop system composed by controller and plant.

Throughout normal operation mode, the system may malfunction. During evaporation, the condenser may fail: the steam can not be cooled and the pressure inside the condenser rises. Therefore, the heater must be switched off to avoid the condenser explosion. By doing so, the temperature of tank1 decreases and the solution may become solid and can not be drained in tank2. Hence, valve V3 must be opened early enough, but after opening first valve V4, for preventing tank 2 overflow.

In the case of a condenser malfunction, we also need to guarantee some response times of the control program, taking into account the timing characteristics of the physical devices:

- whenever a condenser malfunction starts, the condenser can explode if steam is produced during 22 time units;

- if the heating device is switched off, the steam production stops after 12 time units;

- if no steam is produced in tank 1 , the solution may solidify after 19 time units;

- emptying tank 2 takes between 0 and 26 time units;

- emptying tank 1 can be very fast with respect to the other durations, so that it is considered instantaneous;

- filling tank 1 takes at most 6 time units. 


\subsection{Controller specification}

As we use the Simulation and Formal Verification, using different tools and intending to conciliate the obtained results, we adopted a controller specification that is the same for the basis of the controller program in the two analysis techniques. Thus, the controller specification was developed in IEC 60848 SFC because it can be used as the basis for the development of the Programmable Logic Controller program (PLC), to be verified with UPPAAL based on timed automata (Alur and Dill, 1990), and also it is the basis for the controller program to be used by StateGraphs Modelica library (Otter et al. 2005).

The input and output variables of the controller model are summarized on Table 1; minimum and maximum level sensors and malfunction sensor are considered as inputs and on-off valves and Heater, Mixer and Alarm are controller program outputs.

$\underline{\text { Table } 1}$ Input and output variables of the controller program

\begin{tabular}{ll} 
Inputs & Outputs \\
\hline T1F- tank1 full & V1- valve 1 \\
T1E- tank1 empty & V2 - valve 2 \\
T2F - tank2 full & V3 - valve 3 \\
T2E - tank2 empty & V4 - valve 4 \\
Malf - condenser malfunction & H- heater \\
& MR - mixer \\
& Alarm \\
\hline
\end{tabular}

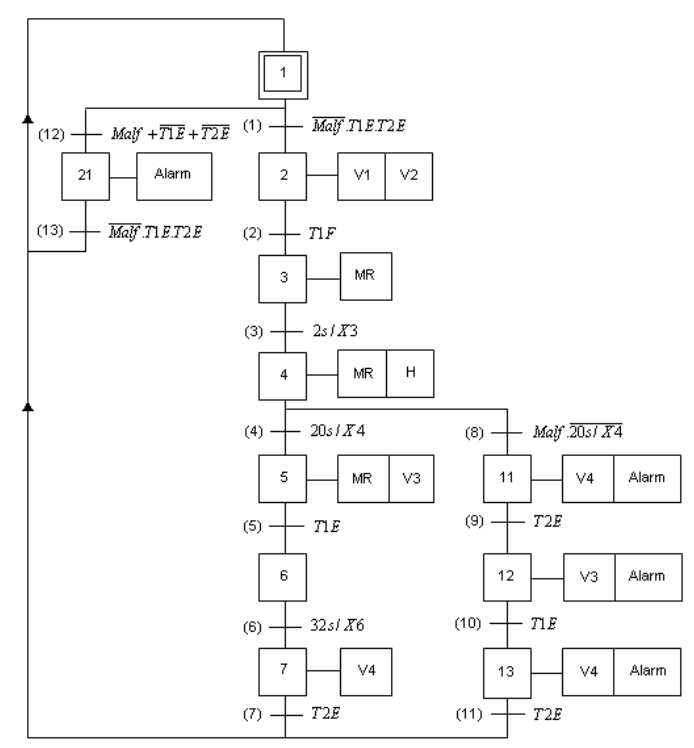

Fig. 2. IEC 60848 SFC specification of the controller program.

In order to guarantee the desired behavior for the described system, a IEC 60848 SFC specification is presented on Fig. 2. As IEC 60848 SFC is a specification language (and not a programming one), it is necessary to translate the SFC specification, first to a StateGraph program, presented in (Seabra et al. 2007) and, second, to translate it into a program written in a PLC programming language (in this case it will be used the ladder language).

\section{PLANT MODELLING}

The interesting point about the plant modeling was that it consisted of two steps: first, to model the plant using Dymola software and Modelica programming language (Elmqvist and Mattson, 1997), and, then, to use the obtained models as a basis for the development of the UPPAAL (David et al. 2003) models which are used on the formal verification tasks. We consider the following eight modules for the plant modeling: Tank1, Tank2, Heater, Mixer, Alarm, Steam, Condenser and Liquid. As this work is devoted to formal verification tasks, only the case of tank1, modeled using Modelica programming Language, and the corresponding UPPAAL model are presented. All the plant is modeled using Modelica programming language in (Seabra et al. 2007). It is remembered that the most important information that is taken into account is the set of simulation functioning delays that are obtained by simulation. These delays are, afterwards, used for the formal verification tasks, in order to define the time units used in the modules of the plant model.

\subsection{Model of tank1}

The model of tank1 is, first, simulated with Dymola, presented as Modelica code in Fig. 3.

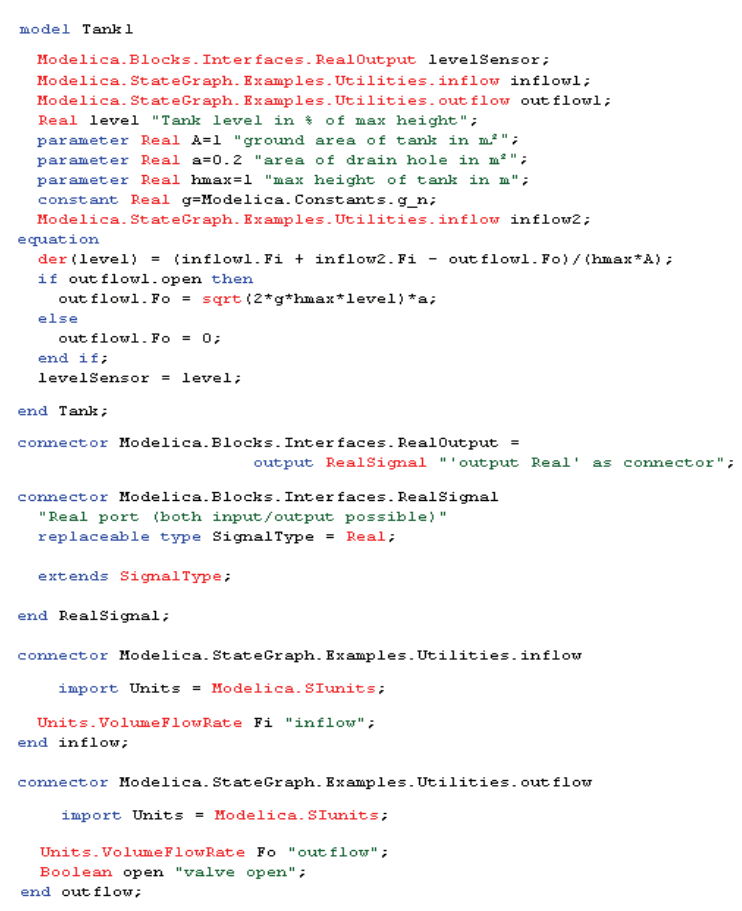

Fig. 3. Modelica code for the tank1 model. 
The obtained delays on simulation were used on formal verification with UPPAAL. The corresponding model of the tank developed in UPPAAL for formal verification purposes is presented in Fig. 4.

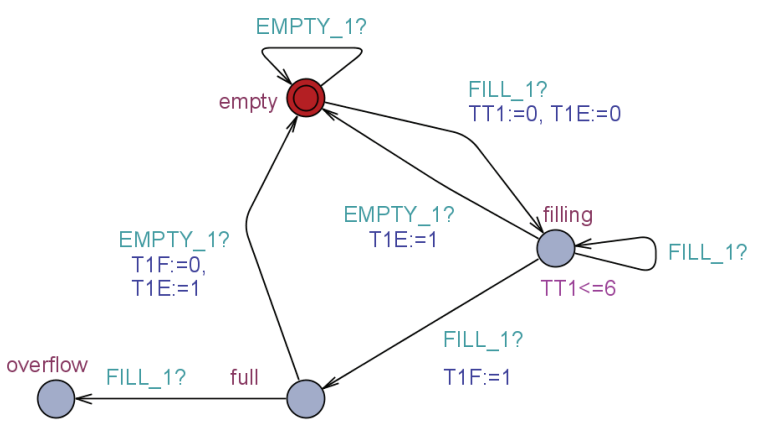

Fig. 4. UPPAAL model of the tank1.

We consider four states: empty models that tank 1 is empty; filling models that the liquid is entering in tank1; full models that tank1 is full; state overflow is also considered, this is a possible state for the tank, but describes an undesired behavior. In this model, it is also considered that the tank 1 is emptied in a very short time, when compared with the filling time. We have considered this time null. It is for that reason that the model goes from the full state directly to the empty state, without an intermediate state. The Boolean variables $\mathrm{T} 1 \mathrm{E}$ and $\mathrm{T} 1 \mathrm{~F}$ are associated with tank1.empty and tank1.full, respectively. These variables represent the level sensors' signals sent by the sensors from the plant to the controller. The maximum time for filling tank 1 is six time units.

\subsection{Model of tank2}

The model of tank 2 is similar at the tank 1 model and the reasoning followed to obtain this model was the same as presented before for obtaining the tank1 model. As empting tank 1 is considered to take a short (null) time, the filling of the tank 2 is done in the same conditions, since the liquid is transferred from tank1 to tank2. Four states are considered: empty, full, emptying and overflow, which is a possible state for the tank, but describes an undesired behavior. The variables T2E and T2F have the same behavior on the tank 2 model as the T1E and T1F described above on the tank1 model. Empty tank 2 takes, at maximum, twenty-six time units.

\subsection{Models of Heater Mixer and Alarm}

The reasoning adopted for building these three models is the same for all of them and consists in considering two states for each one: off (as initial state for all of them) and on that models that respective orders sent from the controller to the plant are actives. In Fig. 5 is presented the model of the mixer, for illustration of all these three models.

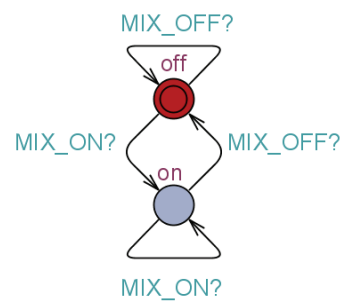

Fig. 5. Model of the mixer

\subsection{Model of the condenser}

The model of the condenser, presented in Fig. 6, is composed by five states: the state good models the good functioning of the condenser; the state malfunction_heater models that the condenser is malfunctioning and the heater is in state on; the state malfunction_not heater models that the condenser is malfunctioning but the heater has been switched off; the state before_explosion models the behavior where it is not possible to avoid the condenser explosion and the state explosion models the behavior of the condenser explosion.

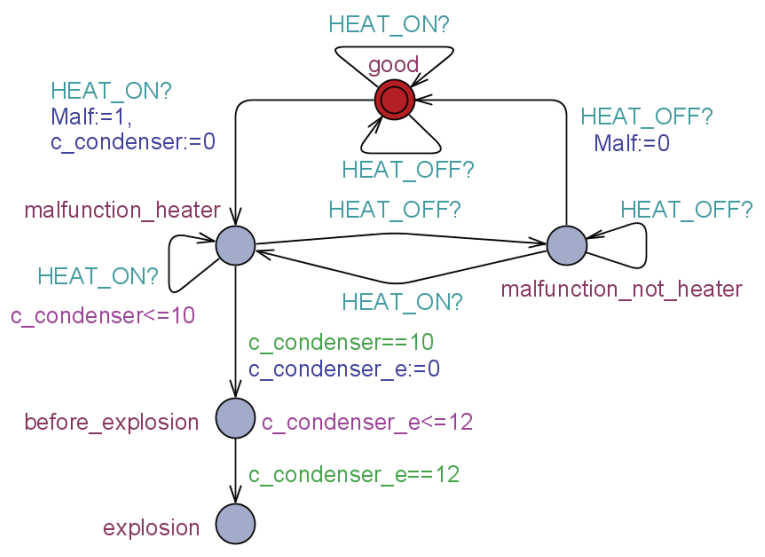

Fig. 6. Model of the condenser

In the behavior described in the case study it is presented that the condenser may explode if it is in malfunctioning behavior and if steam exists for during twenty-two time units after that. In this case, if the system behavior is the same as described in the malfunction_heater state (the heater is in the on state) during ten time units, then the condenser will explode because we will have steam at least for more twelve time units which implies that the condenser will explode (twenty-two time units after). The malfunction behavior happens in a random way from the state good of the condenser model: we have added to the condenser model a Boolean variable Malf that indicates that behavior. 


\subsection{Model of the steam}

One of the hardest tasks on plant modeling is to model plant products (as the case of steam) because these models are specific for each plant. In this case, and taking into account the above described behavior for the system, the model of steam is extremely useful to allow us to prove some system behavior properties. We, thus consider three states for the model: state off models that steam does not exist; state on_heat_on models that steam exists and the heater is also in state on (the model of the steam is dependent of the model of the heater) and state on heat_off models that steam exists but the heater is in the off state. We remember that after the heater switches off, steam exists during twelve time units. We have introduced the Boolean variable steam_var that indicates when steam exists or not.

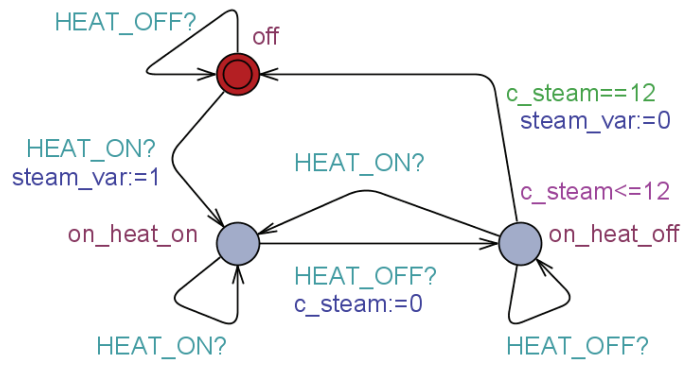

Fig. 7. Model of the steam

\subsection{Model of the liquid}

As the steam model presented before, the model of the liquid is also a specific model related with a plant product.

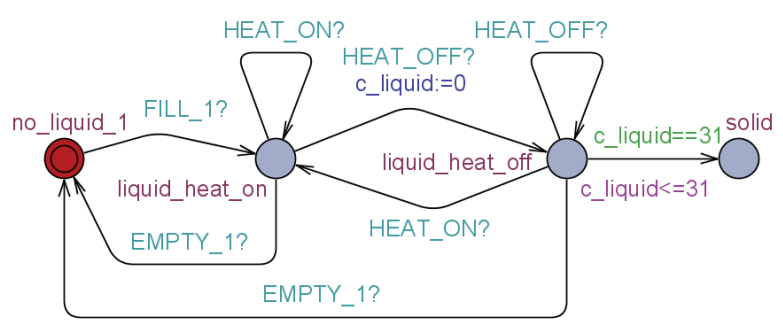

Fig. 8. Model of the liquid

This model has been created from the point of view of the presence of liquid in tank1 to look at its behavior regarding the solidification state of the liquid, possible in tank1. From point of view of the presence of liquid on tank1, Fig. 8, we have three possibilities: there is no liquid in tank 1 , modeled by state no_liquid_1; there is liquid in tank1 and the liquid is heating, modeled by the state liquid_heat_on; there is liquid in tank1 and the liquid is not heating, modeled by state liquid_heat_off; and there is liquid in tank1 that changed to the solid state after thirty-one time units (these thirty-one time units mean that the liquid solidifies after nineteen time units after the absence of steam; we remember that after the heater is switched off, the steam is present for more twelve time units).

We point out that, for all the models, we have adapted the simulation conditions to obtain the same delays proposed on (Gaid et al. 2005), in order to have a basis to compare the formal verification results obtained.

\section{SYSTEM BEHAVIOR PROPERTIES AND PROPERTIES FORMALIZATION}

In order to express the properties in UPPAAL syntax, we need a small part of TCTL formalism (Alur et al., 1993). In the formulas below which are all (possibly timed) invariants, $A$ is the universal quantifier on paths: for any path..., and [ ] means always... The combination A [ ] means for all states in the future...

The properties that we intend to prove are:

- P1: To prevent tank 1 from damage by overheating, it must be checked that the tank is full when the heater is working.

- P2: During the evaporation step, steam can leave tank 1 into the condenser only when the heater is on and the valves V1, V2 and V3 are closed.

- P3: To prevent uncontrolled liquid flow, the input and output valves of a tank must not be simultaneously open.

- P4: Condenser must not explode.

- P5: Solution must not solidify in tank 1.

- P6: Tank1 must not overflow.

- P7: Tank2 must not overflow.

The properties are formalized as follows, using TCTL:

- P1: A[ ] (H imply T1F);

- P2: A[ ] (H imply (not (V1) and not (V2) and not (V3)));

- P3: A[ ] not (((V1 || V2) and V3) or (V3 and $\mathrm{V} 4)$ );

- P4: A[ ] not condenser.explosion

- P5: A[ ] not liquid.solid

- P6: A[ ] not tank1.overflow

- P7: A[ ] not tank2.overflow

\section{FORMAL VERIFICATION}

The adopted strategy for formal verification considers that the program is written in Ladder language (according section 2) and some work hypotheses, as presented on (Gaid et al. 2005):

- the PLC executes the control program with a three phases cyclic behavior;

- there are considered timer blocks IEC 61131-3 TON;

- the duration of PLC cycle is considered to be between $\varepsilon_{1}$ and $\varepsilon_{2}$ and we have adopted $\varepsilon_{1}$ 
equal to 0 and $\varepsilon_{2}$ equal to 1 ;

- the unit of time used for the plant is irrelevant;

- discovering the maximal value of $\varepsilon_{2}$ for the properties to hold was not our objective.

The controller and the plant models, coupled as a closed-loop system, compose the global model verified. All the properties were verified in less than 2 seconds each, using. UPPAAL version 4.0.3 and an Intel Core Duo, $1,87 \mathrm{GHz}$, machine with $4 \mathrm{~GB}$ of RAM. The modular approach to building the plant model is well suited to facilitate the tasks of writing the properties, as is the case of properties P4, P5, P6 and P7.

\section{CONCLUSIONS AND FUTURE WORK}

The presented way to build plant models, from an automation system, is useful for two important points: first, we can avoid, using simulation, a set of program errors in reduced time intervals which would not happen if these errors were detected only through the use of formal verification techniques and, second, our modular approach allow us an easier way to build plant models which is well suited to facilitate the tasks of writing the properties formulae. The consideration of undesirable states on the created plant models allows us an easier writing of properties.

The use of Modelica programming language, to obtain these modular plant models, is useful to define the delays in which a property can, or cannot, be proved and the delays to elaborate the considered plant models.

The simulation techniques allow us to test different delays of the plant functioning and to see if a property, for different considered delays, is still true or if different delays imply that a property that is true, for a delay, will become false for another. This study is not presented in this work but it is very important from the point of view of system safety.

\section{REFERENCES}

Alur R., C. Courcoubetis and D. L. Dill (1993). Model-Checking in Dense Real-Time, Information and Computation, vol. 104, $\mathrm{n}_{-} 1, \mathrm{pp}$. 2-34.

Alur R. and D. L. Dill (1990),,. Automata for modeling real-time systems. Proc. of 17 th Int. Coll. Automata, Languages, and Programming (ICALP'90), England.

Baresi L., M. Mauri, A. Monti and M. Pezzè (2000). PLCTOOLS: Design, Formal Validation, and Code Generation for Programmable Controllers. Simulation software.

David A., G. Behrmann, K. G. Larsen and W. Yi (2003). A Tool Architecture for the Next
Generation of UpPAAL, Technical Report n. 2003011, Uppsala University, February. 20 pages.

Elmqvist E. and S. Mattson (1997). An Introduction to the Physical Modelling Language Modelica. Proceedings of the 9th European Simulation Symposium, ESS'97, Oct 19-23, Passau, Germany.

Gaid M., B. Bérard and O. Smet (2005). Verification of an evaporator system with UPPAAL. European Journal of Automated Systems, vol. 39, nº9, pp. 1079-1098.

Huuck R., B. Lukoschus and Y. Lakhnech (2001). Verifying Untimed and Timed Aspects of the Experimental Batch Plant. European Journal of Control, vol. 7, n_4, p. 400-415.

Kowalewski S., O. Stursberg and N. Bauer (2001). An Experimental Batch Plant as a Test Case for the Verication of Hybrid Systems. European Journal of Control. vol. 7, n_4, pp. 400-415.

Machado J., B. Denis, J.-J. Lesage (2006). A generic approach to build plant models for DES verification purposes. Proc. of Wodes'2006 $-8^{\text {th }}$ Workshop on Discrete Event Sistems. July, 1012, Ann Arbor, Michigan, USA.

Mader A. and H. Wupper (1999). Timed Automaton Models for Simple Programmable Logic Controllers. Proc. Of the 11th Euromicro Conference on Real-Time Systems (ECRTS'99), York, UK.

Moon I. (1994). Modeling programmable logic controllers for logic verification. IEEE Control Systems, vol. 14, nº2, pp. 53-59.

Otter M., K. Årzén and I. Dressler (2005). StateGraph - A Modelica Library for Hierarchical State Machines. In: Modelica 2005 Proceedings.

Remelhe M., S. Lohmann, O. Stursberg and S. Engell (2004). Algorithmic Verification of logic Controllers given as Sequential Function Charts. Proceedings of the IEEE Intemational Symposium on Computer Aided Control Systems Design, Taipei, Taiwan.

Roussel J-M. and B. Denis. (2002). Safety properties verification of ladder diagram programs. European Journal of Automated Systems, Vol. 36, pp. 905-917.

Rossi O. (2004). Validation formelle de programmes ladder pour automates programmables industriels. PhD thesis, June, France.

Seabra E., J. Machado, F. Soares, C. Leão and J. Silva (2007). Simulation and formal verification of real-time systems: A case study. In: ICINCO'2007 proceedings, 9-12 $2^{\text {th }}$ may, Angers, France. 